\title{
Sonoluminescence, Shock Waves, and Micro-Thermonuclear Fusion
}

\author{
W.C. Moss \\ D.B. Clarke \\ J.W. White \\ D.A. Young \\ For presentation at the 1995 APS Topical Conference on \\ Shock Compression of Condensed Matter \\ Seattle, WA, August 13-18, 1995
}

\section{August 1995}

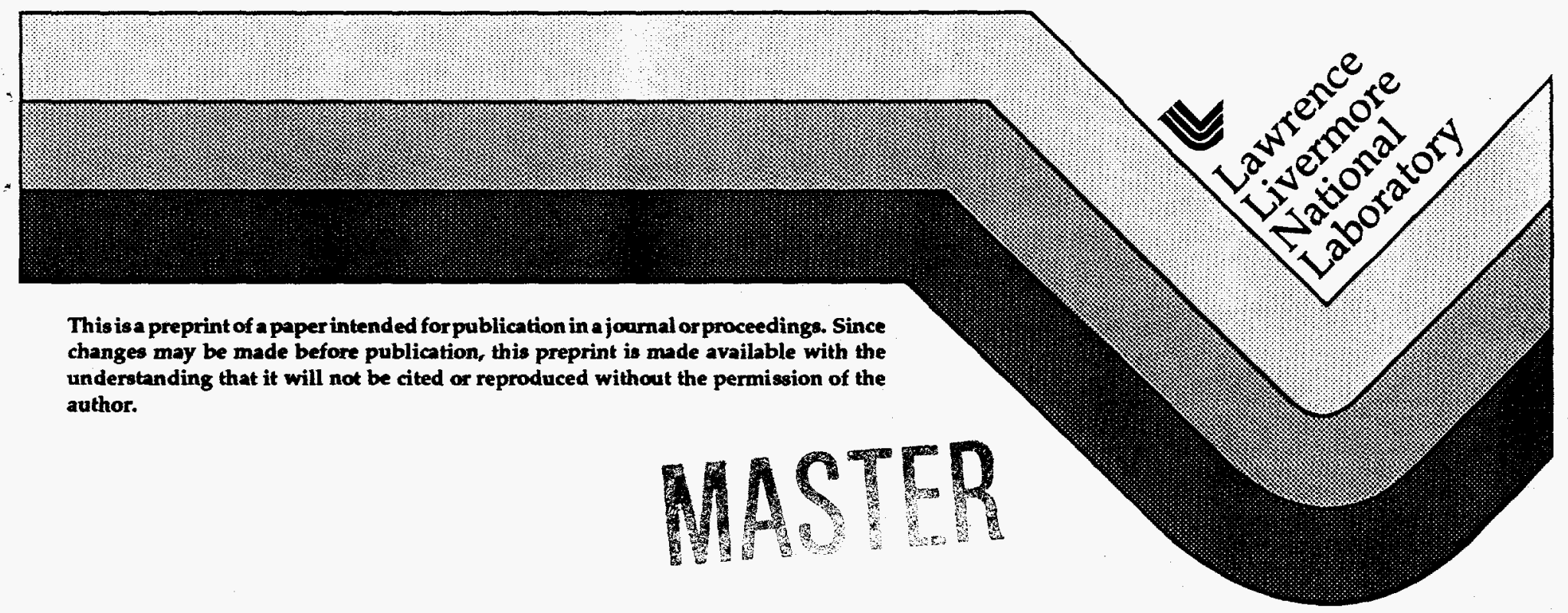

This is a preprint of a paper intended for publication in a joumal or proceedings. Since changes may be made before publication, this preprint is made available with the understanding that it will not be cited or reproduced without the permission of the author. 


\section{DISCLAIMER}

Portions of this document may be illegible in electronic image products. Images are produced from the best available original document. 


\title{
SONOLUMINESCENCE, SHOCK WAVES, AND MICRO-THERMONUCLEAR FUSION
}

\author{
W. C. Moss, D. B. Clarke, J. W. White, and D. A. Young \\ Lawrence Livermore National Laboratory, PO Box 808, Livermore, CA 94550
}

\begin{abstract}
We have performed numerical hydrodynamic simulations of the growth and collapse of a sonoluminescing bubble in a liquid. Our calculations show that spherically converging shock waves are generated during the collapse of the bubble. The combination of the shock waves and a realistic equation of state for the gas in the bubble provides an explanation for the measured picosecond optical pulse widths and indicates that the temperatures near the center of the bubble may exceed $30 \mathrm{eV}$. This leads naturally to speculation about obtaining micro-thermonuclear fusion in a bubble filled with deuterium $\left(D_{2}\right)$ gas. Consequently, we performed numerical simulations of the collapse of a $D_{2}$ bubble in $D_{2} O$. A pressure spike added to the periodic driving amplitude creates temperatures that may be sufficient to generate a very small, but measurable number of thermonuclear D-D fusion reactions in the bubble.
\end{abstract}

\section{INTRODUCTION}

Sonoluminescence involves the conversion of acoustical energy to optical energy. It arises from the nucleation, growth, and collapse of gas-filled bubbles in a liquid. There are two main types of sonoluminescence: single bubble and multiple bubble. We will discuss single bubble sonoluminescence (SBSL) in this paper. Figure 1 shows a typical SBSL experimental configuration. A spherical flask that has a radius of a few centimeters contains a degassed liquid, usually water or a glycerine-water mixture. Acoustic transducers placed around the outside of the flask provide an oscillatory driving pressure that creates standing waves in the liquid. The driving frequency is typically near the fundamental radial mode of the flask/liquid system, e.g., $\approx 27 \mathrm{kHz}$ for a $3 \mathrm{~cm}$ radius flask. A gas bubble inserted into the liquid is trapped (levitated) near the center of the flask and oscillates radially. At sufficiently high transducer amplitudes, the compressed gas radiates optically, at the conclusion of the collapse phase.

Sonoluminescence was discovered in 1933 by Marinesco and Trillat, ${ }^{1}$ who observed that photo-

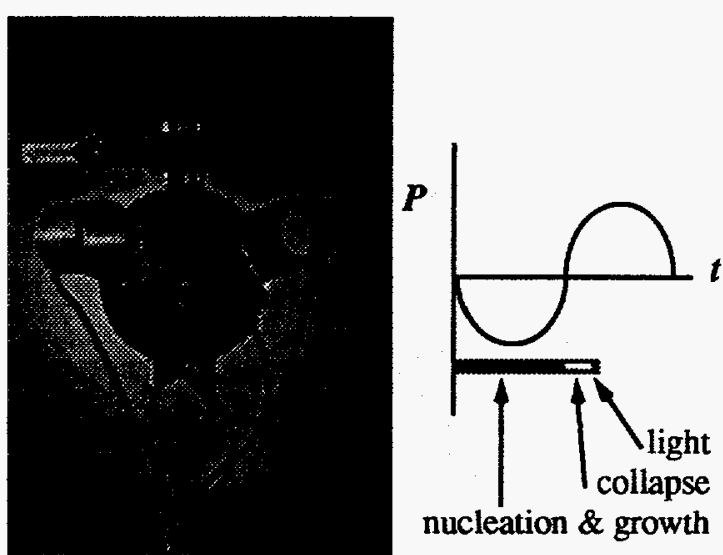

FIGURE 1. Typical SBSL experimental configuration. The flask is surrounded by four acoustic transducers. The graph shows qualitatively the regions of bubble growth, collapse, and light emission as a function of the pressure at the liquid-gas interface

graphic plates became exposed when the developing emulsion was agitated with ultrasound. Frenzel and Schultes, ${ }^{2}$ hypothesized that the exposure was due to light. In 1959, Meyer and Kuttruff ${ }^{3}$ photographed sonoluminescing bubbles that were grown and collapsed on the end of a nickel rod. They established 
that the light is emitted as a discrete flash with the periodicity of the driving sound field, as shown in Fig. 1. During the next 30 years, experiments showed that the optical emission was due to heat in the collapsing bubbles, although the exact mechanism(s) was unclear. In 1990, D. F. Gaitan ${ }^{4,5}$ discovered the method described in the preceding paragraph for acoustically levitating a single sonoluminescing bubble. This remarkable achievement allowed researchers to study systematically the physics of sonoluminescence and set the stage for a surprising observation.

Using Gaitan's method, Barber and Putterman ${ }^{6}$ reported emission spectra from a single pulsating bubble that were synchronous with the driving (oscillatory) acoustic field, and had a measured pulse width $\leq 50 \mathrm{ps}$. The emission spectra were consistent with $\mathrm{a} \geq 2 \mathrm{eV}$ black body radiator. ${ }^{7}$ The underlying physics of the short pulse widths and the inferred high temperatures could not be explained by any current theory of sonoluminescence.

A plausible explanation is that picosecond sonoluminescence is the result of two mechanisms; The first mechanism heats the gas rapidly, which initiates optical emission, and is followed immediately by the second mechanism, which quenches the emissions. We hypothesized that rapid heating by a spherically convergent shock and rapid cooling by spherically divergent flow could explain the observed pulse widths, if they could occur rapidly enough. ${ }^{8}$ Prior to our work, the full hydrodynamic details (nonlinearly compressible liquid, high pressure and temperature gas equation of state, and consistent boundary conditions) of the late time collapse of a bubble had not been studied. The most complete earlier analysis was performed by $\mathrm{Wu}$ and Roberts, ${ }^{9}$ who considered the hydrodynamics of a van der Waals gas, with a constant specific heat. The motion of the liquid-gas interface was obtained from the Rayleigh-Plesset ${ }^{10-13}$ equation, which models the liquid as an almost incompressible fluid. Although their analysis showed the development of a spherically convergent shock, they did not determine the underlying physics of the picosecond pulse widths. In addition, their $1 \mathrm{keV}$ calculated temperature at the center of the bubble is well beyond the range of validity of a constant specific heat van der
Waals gas equation of state. Consequently, we performed more detailed analyses that include realistic gas equations of state and the nonlinear hydrodynamics of the liquid that surrounds the bubble.

\section{AIR-WATER NUMERICAL SIMULATIONS}

We assume spherical symmetry and consider the motion of a gas-filled bubble with initial radius $R_{o}$ surrounded by a shell of water, whose outer radius is $R_{W}$. The gas and water are initially at atmospheric pressure, $P_{o}=1 \mathrm{bar}$, in thermal equilibrium, and at rest. The outer radius of the water is driven by an oscillatory pressure $P_{o}-P_{a} \sin \omega t$. We calculate the bubble's response to only one cycle of the driving oscillatory pressure, that is, only one of the many growth and collapse cycles that the bubble experiences. We assume the physics that governs the creation of any one of the steady state sonoluminescent flashes can be approximated adequately using typical values for $R_{o}, R_{W}, \omega$, and $P_{a}$, because the bubble collapse is primarily an inertial effect of the liquid compressing the gas. Any set of parameters that produces a typical bubble radius, as a function of time, should be sufficient to supply the necessary inertial forces that generate the flash. We have chosen parameters that are typical, but not representative of any particular experiment. We used $R_{o}=10 \mu \mathrm{m}$, $R_{W}=3 \mathrm{~cm}$, and $\omega=2 \pi(27.6 \mathrm{kHz}) .{ }^{14}$ The pressure amplitude $P_{a}=0.4$ bar was chosen to yield a maximum bubble radius of $\approx 90 \mu \mathrm{m}$, so the ratio $R_{\max } / R_{0}$ is consistent with experimental data. ${ }^{15}$

In the absence of viscosity, surface tension, heat conduction, mass diffusion, and nonmechanical energy loss (radiation), the equations for the conservation of mass, momentum, and energy for the system are ${ }^{16,17}$

$$
\begin{aligned}
\frac{D \rho}{D t}+\rho \nabla \bullet \mathbf{v} & =0, \\
\rho \frac{D \mathbf{v}}{D t}+\nabla(P+Q) & =0, \quad \text { and } \\
\frac{D \varepsilon}{D t}+(P+Q) \frac{D V}{D t} & =0,
\end{aligned}
$$


where $\quad \rho, \mathbf{v}, Q\left(|\nabla \mathbf{v}|^{2}\right), P(\rho, T), \varepsilon(\rho, T)$, and $V$ are the density, velocity, artificial viscosity, ${ }^{17}$ pressure, specific intemal energy, and specific volume $\left[\rho^{-1}\right]$, and $\frac{D}{D t} \equiv \frac{\partial}{\partial t}+\mathrm{v} \bullet \nabla$. Artificial viscosity is a standard computational method that gives shocks a finite thickness, so infinite gradients do not develop. The water was described by a polynomial equation of state. ${ }^{18}$ The air was described by an analytic model $^{8}$ that includes vibrational excitation, dissociation, ionization, and repulsive and atractive intermolecular potentials. It was constructed from a combination of data and theory and is believed to be valid for the domains of density and temperature in our calculations.

The equations of motion [Eq. (1)] and the equations of state, combined with the boundary and initial conditions given above comprise a complete set of equations that can be solved for the radial and temporal variation of all the field quantities. We solved numerically the differential equations incrementally in time, using a finite difference method. The water and the gas are divided into a mesh consisting of many concentric shells (zones) of fixed mass. The mass may vary from one shell to another. There must be enough shells to ensure that the finite difference solution converges to the differential equation solution.

Figure 2 shows the time dependence of the bub-

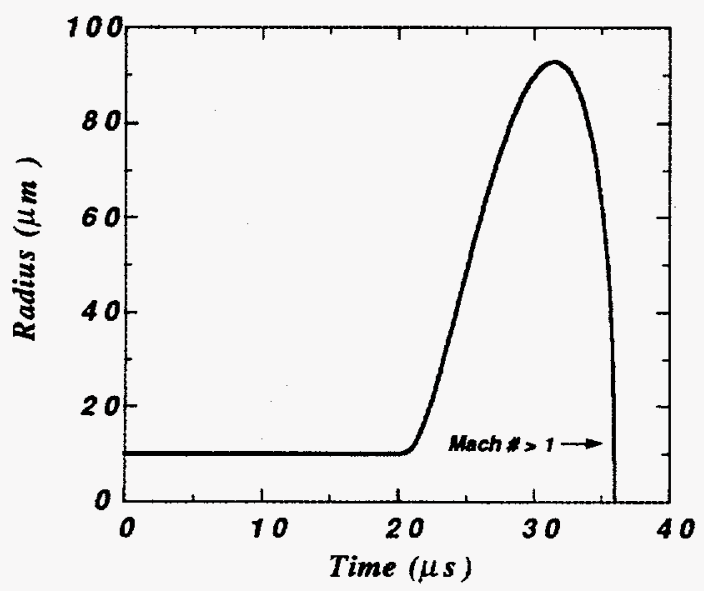

FIGURE 2. Calculated $R(t)$ for an air bubble in water. ble radius $R$, for the conditions above. It takes $\approx 22 \mu \mathrm{s}$ for the initial rarefaction to travel from $R_{W}$ to $R_{o}$, after which, the bubble begins to grow. The collapse velocity becomes supersonic ( $\dot{R}$ / sound speed at center of bubble) when $R=12 \mu \mathrm{m}$, which generates one or more spherically converging shock waves. ${ }^{19}$ Figure 3 shows the time dependence of the

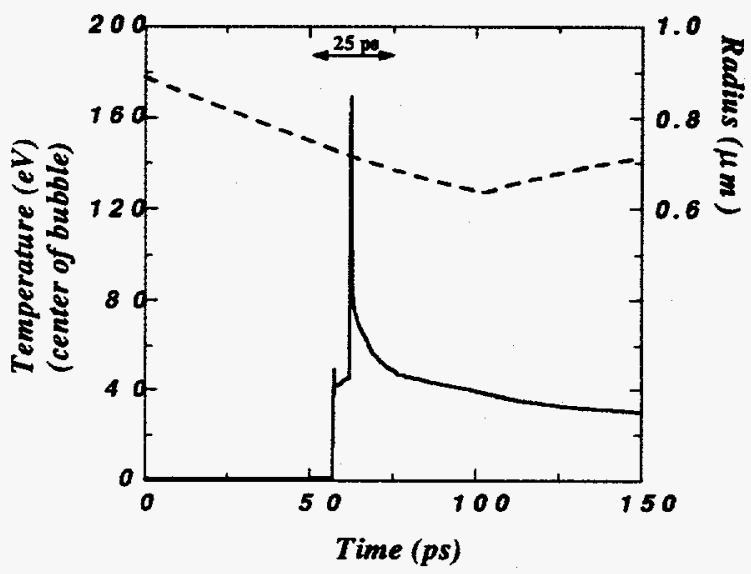

FIGURE 3. Calculated time dependence of the temperature (solid line) at the center of the air bubble when the shock waves reflect from the center of the bubble. The dashed line shows $R(t)$.

temperature at the center of the bubble when the shock waves reflect from the center of the bubble. The dashed line shows $R(t)$. The shock waves reach the center (creating high temperatures) before the bubble reaches its minimum radius. The reflection of the shocks from the center of the bubble quenches the collapse. The peak temperature exceeds $100 \mathrm{eV}$. We compute an optical pulse width of $5 \mathrm{ps}$ and a total radiated power of $0.4 \mathrm{~W}$, using the calculated $T(r, t)$ and assuming that the bubble radiates as an optically thin black body, ${ }^{8}$ which is an adequate approximation for this discussion. These results are consistent with experimental measurements of the pulse width and the radiated power. ${ }^{6,7}$ These results show that sufficient mechanical energy can be delivered in a short enough time to account for picosecond sonoluminescence.

The main physics results of our air-water simulations can be summarized as follows. Three mecha- 
nisms control the pulse width and the temperature at the center of the bubble: shock waves, the gas intermolecular potential, and ionization. Shock waves and a realistic representation of the gas intermolecular potential are required to generate picosecond sonoluminescence. The pulse width increases to $\approx 150 \mathrm{ps}$, if the intermolecular potential is unrealistically soft, as in an ideal gas. The pulse width is $\approx 500 \mathrm{ps}$ if no shock is present. The temperature of the bubble increases as it collapses, due to $P d V$ work. The increased specific heat due to ionization mitigates the high temperatures at the center of the bubble, because it removes energy that would otherwise be used to heat the gas. Simulations without ionization produced $700 \mathrm{eV}$ temperatures at the center of the bubble, compared to the $100 \mathrm{eV}$ temperatures with ionization.

The effect of ionization on the peak temperature raises an interesting possibility: air (nitrogen) has seven electrons, hydrogen has 1 electron. Consider a bubble of hydrogen or deuterium instead of air. After the one electron is ionized, the remaining energy will heat the gas. Is it possible to heat the deuterium enough for micro-thermonuclear fusion in a deuterium gas and deuterated water system? Temperatures $\geq 0.5 \mathrm{keV}$ are required.

\section{$D_{2}-D_{2} O$ NUMERICAL SIMULATIONS}

Figure 4 (dashed line, scale on right) shows the peak temperature at the center of a deuterium bubble in deuterated water. ${ }^{20}$ The initial and boundary conditions are identical to the to air-water simulation. We used appropriate equations of state for the gas and the liquid. ${ }^{20}$ The figure shows a peak temperature of $\approx 0.5 \mathrm{eV}$ and $\mathrm{a} \approx 500 \mathrm{ps}$ pulse width. These results are inconsistent with experimental data, ${ }^{21}$ which showed measured pulse widths less than $15 \mathrm{ps}$ and violet-colored visible emissions. The violet color suggests central temperatures that are an order of magnitude higher than those calculated.

The discrepancy can be explained by modelling the contents of the bubble differently. The bubble does not contain only pure deuterium, it also contains deuterated water vapor. The short measured pulse width and our modelling of the air-water system suggest that shocks are present. The added mass

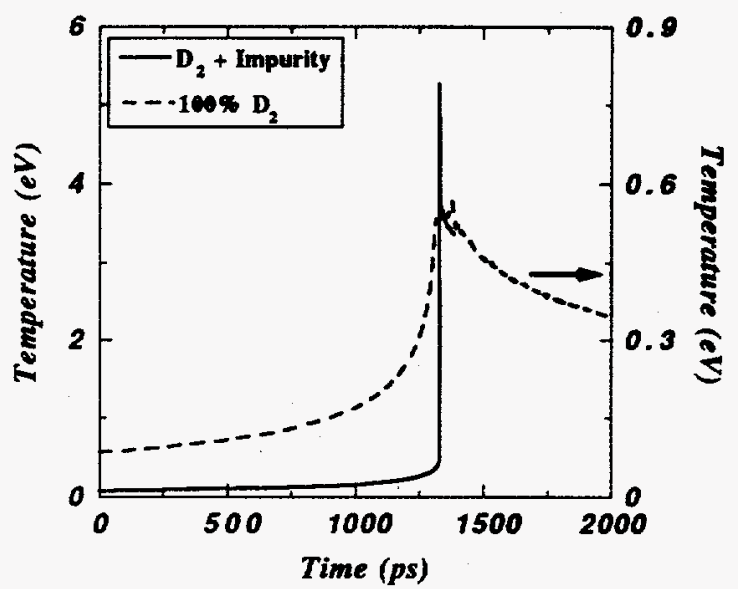

FIGURE 4. Peak temperatures at the center of a bubble containing pure $\mathrm{D}_{2}$ (dashed line) and a $15 \%$ molar impurity $\left[\approx \mathrm{D}_{2} \mathrm{O}\right.$ vapor] (solid line). The vapor lowers the sound speed, which makes shock generation easier. The calculated pulse width and peak temperature of the mixture are consistent with experimental data.

of the vapor lowers the sound speed $(c=\sqrt{\gamma P / p})$ of the mixture, which makes it easier to shock. We do not know how to estimate the water vapor fraction, nor do we have an equation of state for water that is valid for rarified vapor, condensate, and compressed dense liquid. Consequently, we use our air equation of state, which is valid in all these regions. It is reasonable to approximate the water vapor using an air equation of state, because we are interested primarily in the sound speed reduction due to added mass. Figure 4 (solid line, left scale) shows the calculated time dependence of the temperature at the center of a collapsing bubble, which contains a mixture of $85 \%$ molar deuterium and $15 \%$ molar air ( $\approx$ deuterated water vapor). The sound speed of the mixture at STP is $657 \mathrm{~m} / \mathrm{s}$, versus $913 \mathrm{~m} / \mathrm{s}$ for pure deuterium. The pulse width and temperature are now more consistent with the experimental data, and these results are insensitive to small changes in the molar ratios.

\section{Methods to Enhance the Implosion}

The consistency of the deuterium+vapor simulation with the experimental data provides a starting point for examining practical methods to enhance 
the implosion. There are many parameters to vary, including the flask (size, thickness, material), liquid composition, bubble size, ambient temperature and pressure, and driving pressure (frequency, structure, and amplitude). The most practical approach is to minimize the perturbations to a standard sonoluminescing system. Consequently, we would like to identify the simplest perturbation that has a large influence. We consider pulse-shaping the driving amplitude. Figure 5 (inset) shows a 5 bar triangular spike superimposed on the $1.0 \pm 0.4 \mathrm{bar} 27.6 \mathrm{kHz}$ driving pressure. The center of the spike is at $16.5 \mu \mathrm{s}$, and its base width is $0.5 \mu \mathrm{s}$. The sinusoidal drive is responsible for establishing the gross system acoustics, to which sonoluminescence is extremely sensitive. We hypothesize that the spike can supply energy without affecting the mechanisms that allow sonoluminescence to occur. Preliminary experimental data ${ }^{21}$ show that a deuterium bubble is brighter when the driving amplitude has a spike. Figure 5

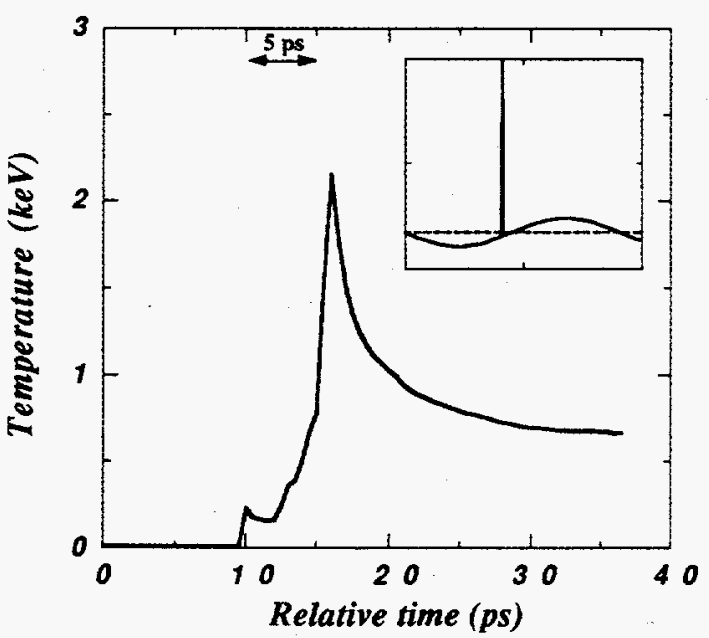

FIGURE 5. The inset shows the time dependence $(40 \mu$ s full scale) of one cycle of the flask driving pressure ( 6 bar full scale). A 5 bar triangular spike is superimposed on the sinusoid. The main figure shows the peak temperature at the center of the bubble due to the spiked drive.

shows the calculated time dependence of the temperature at the center of the bubble, due to the driving pressure shown in the inset. The peak central temperature occurs $11 \mathrm{ps}$ before the bubble reaches its minimum radius (not shown in the figure). The spike accelerates the interface to supersonic velocities much earlier in the collapse than in the previous simulations. This provides ample time and distance for spherical convergence to increase greatly the amplitude of the shocks. In general, the central temperature increases, as the spike amplitude increases, or the center of the spike occurs later in time, or the base width of the spike decreases.

Figure 6 (solid line) shows the peak temperature

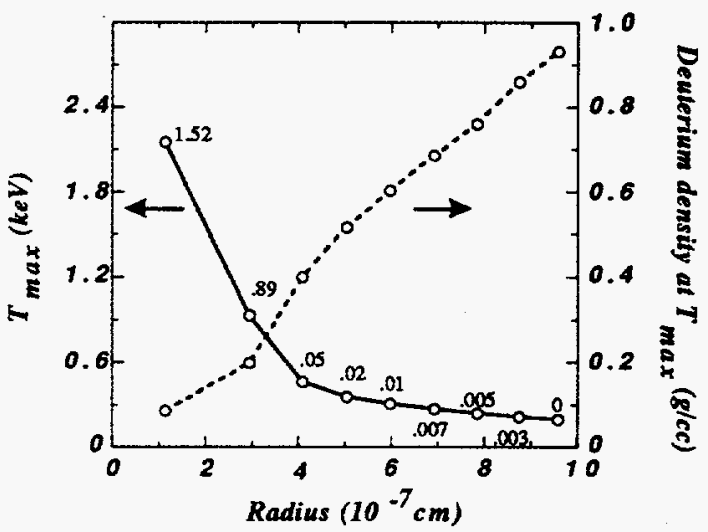

FIGURE 6. Calculated peak temperature (solid line) and $D_{2}$ density (dashed line) at the centers (circles) of the innermost nine computational zones. The numbers along the solid line are calculated number of neutrons per hour from each zone.

at the center of the innermost nine zones (indicated by circles). The dashed line shows the deuterium density (at the peak temperature) at the same locations. We excluded thermal conduction and radiant energy transport from our simulations, to simplify the analysis of the complex hydrodynamic effects on the implosion due to the spike. The effects of thermal conduction and radiant energy transport become important typically when temperatures exceed $0.5 \mathrm{keV}$, so the calculated temperatures in the innermost couple of zones somewhat over-estimate the actual values. These energy transport mechanisms must be considered in subsequent analyses.

We may obtain a conservative estimate of the number of neutrons produced by the thermonuclear reaction $\mathrm{D}+\mathrm{D} \rightarrow{ }^{3} \mathrm{He}+\mathbf{n}+3.2 \mathrm{MeV}$, if we ignore the innermost two zones and the energy flux from these zones into the adjacent ones. Using Fig. 6 and the formula ${ }^{22} \dot{N}=0.5 n^{2} \overline{\sigma v}$, where $\dot{N}, n$, and 
$\overline{\sigma v}$ are the number of neutrons/(cc-s), the number of deuterons/cc, and the velocity-weighted $D-D$ reaction cross section, ${ }^{23}$ we integrate over the reaction volume and flash duration, then multiply by the number of flashes per hour $(27,600 \times 3600)$. Figure 6 shows the resulting neutron production rates per hour in each of the zones. We calculate a total rate of 0.1 neutron/hour, neglecting the innermost two zones.

Although the count rate is low, it should be measurable. We assume that the neutron production is coincident with the flash, so the time of arrival of the neutrons at the detector can be determined accurately. Furthermore, the energy spectrum of the neutrons is also well-defined, so temporal and energy gating of the detector can remove most of the spurious background signals. ${ }^{21}$ We still expect to obtain a measurable signal, even after including the reductions due to the efficiency and the solid angle of the detector.

Our simulations provide the foundation for a serious attempt to attain thermonuclear fusion from a sonoluminescing bubble. Although the predicted count rate is small, it is interesting scientifically. The main point we want to emphasize is that the spiked drive delivers a lot more energy to the center of the bubble than a purely sinusoidal drive. Consequently, our simulations indicate that the spiked drive may have the potential to supply enough energy to generate a small amount of fusion. The parameter space is large, and there are many ways to enhance the theoretical estimates. For example, the neutron rate can be increased by at least a factor of 50 if the deuterium is replaced by a mixture of deuterium and tritium. ${ }^{23}$ Further modifications to the spike amplitude, timing, and shape may also provide enhancements. Raising the ambient pressure to increase the bubble mass may also be beneficial. At the very least, the spiked driving pressure applied to any sonoluminescing system may produce an experimental crucible that gives the general scientific community easy and inexpensive access to pressures, temperatures, and time scales that have been unattainable previously.

\section{ACKNOWLEDGMENTS}

This work was performed under the auspices of the U. S. Department of Energy by Lawrence Livermore National Laboratory under Contract No. W-7405-Eng-48.

\section{REFERENCES}

1. Marinesco, M., and Trillat, J. J., Comptes Rendus Acad. Sci., 196, 858 (1933).

2. Frenzel, J., and Schultes, H., Zeit. fur Phys. Chem., B27, 421 (1934).

3. Meyer, E., and Kuttruff, H., Zeit. angew. Phys., 11, 325 (1959).

4. Gaitan, D. F., "An experimental investigation of acoustic cavitation in gaseous liquids," Ph. D. dissertation, Univ. of Miss., (1990).

5. Gaitan, D. F., Crum, L. A., Church, C. C., and Roy, R. A., J. Acoust. Sac. Am., 91, 3166 (1992).

6. Barber, B. P., and Putterman, S. J., Nature, 352, 318 (1991).

7. Hiller, R., and Putterman, S. J., and Barber, B. P., Phys. Rev. Lett., 69, 1182 (1992).

8. Moss, W. C., Clarke, D. B., White, J. W., and Young, D. A., Phys. Fluids, 6, 2979 (1994).

9. Wu, C. C., and Roberts, P. H., Phys. Rev. Lett., 70, 3424 (1993).

10. Lord Rayleigh, Phil. Mag., 34, 94 (1917).

11. Noltingk, B. E., and Neppiras, E. A., Proc. Phys. Soc. (London), B63, 674 (1950).

12. Plesset, M., J. Appl. Mech., 16, 277 (1949).

13. Keller, J. B., and Miskis, M., J. Acoust. Soc. Am., 68, 628 (1980).

14. Sweider, D., (LLNL) private communication.

15. Barber, B. P., and Putterman, S. J., Phys. Rev. Lett., 69, 3839 (1992).

16. Zel'dovich, Ya. B., and Raizer, Yu. P., Physics of Shock Waves and High-Temperature Hydrodynamic Phenomena, (Academic Press, 1966), ch. I, II.

17. von Neumann, J., and Richtmeyer, R. D., J. Appl. Phys., 21, 232 (1950)

18. Ree, F. H., LLNL Report UCRL-52190 (1976).

19. See Ref. 8., Fig. 4 and Fig. 7.

20. Moss, W. C., Clarke, D. B., White, J. W., and Young, D. A., LLNL Report UCRL-JC-120422 (1995).

21. Moran, M., et al., LLNL, unpublished.

22. Glasstone, S., and Lovberg, R. H., Controlled Thermonuclear Reactions, (van Nostrand Co., Inc., Princeton, 1960), ch. 2.

23. Bosch, H. -S., and Hale, G. M., Nuclear Fusion, 32, 611 (1992). 